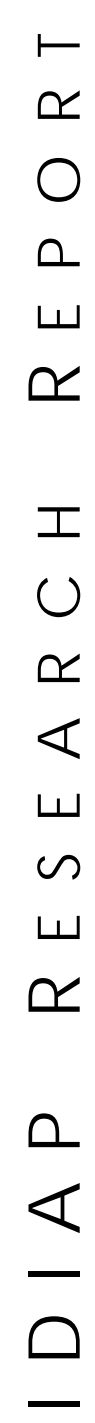

ш

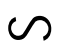

ш

ฯ

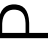

$\varangle$

alle Molle Institute for Perceptual Artificial Intelligence • P.O.Box 592 •

Martigny $\bullet$ Valais $\bullet$ Switzerland

phone $+41-27-721 \quad 77 \quad 11$

fax $\quad+41-27-721 \quad 77 \quad 12$

e-mail secretariat@idiap.ch internet http://www.idiap.ch

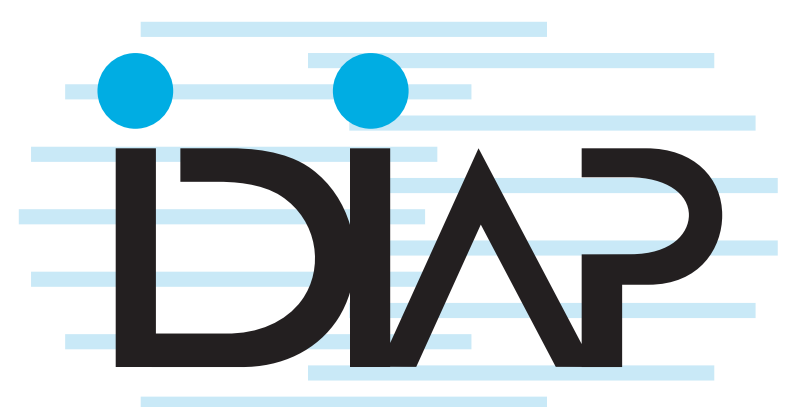

\section{A Parallel Mixture of SVMS FOR VERY LARGE SCALE PROBLEMS}

\author{
Ronan Collobert ${ }^{\mathrm{a}}{ }^{\mathrm{b}} \quad$ Samy Bengio ${ }^{\mathrm{a}}$ \\ Yoshua Bengio ${ }^{b}$ \\ IDIAP-RR 01-12
}

APRIL 26, 2002

PUBLISHED IN

Neural Computation, vol 14, number 5, 2002

\footnotetext{
a IDIAP, CP 592, 1920 Martigny, Switzerland, \{collober, bengio\}@idiap.ch

b Université de Montréal, DIRO, CP 6128, Succ. Centre-Ville, Montréal, Québec,
} Canada, \{collober, bengioy\}@iro.umontreal.ca 

IDIAP Research Report 01-12

\title{
A Parallel Mixture of SVMs for Very Large SCALE PROBLEMS
}

\author{
Ronan Collobert \\ Samy Bengio \\ Yoshua Bengio
}

APRIL 26, 2002

PUBLISHED IN

Neural Computation, vol 14, number 5, 2002

\begin{abstract}
Support Vector Machines (SVMs) are currently the state-of-the-art models for many classification problems but they suffer from the complexity of their training algorithm which is at least quadratic with respect to the number of examples. Hence, it is hopeless to try to solve real-life problems having more than a few hundreds of thousands examples with SVMs. The present paper proposes a new mixture of SVMs that can be easily implemented in parallel and where each SVM is trained on a small subset of the whole dataset. Experiments on a large benchmark dataset (Forest) yielded significant time improvement (time complexity appears empirically to locally grow linearly with the number of examples). In addition, and that is a surprise, a significant improvement in generalization was observed.
\end{abstract}




\section{Introduction}

Recently a lot of work has been done around Support Vector Machines (Vapnik, 1995), mainly due to their impressive generalization performances on classification problems when compared to other algorithms such as artificial neural networks (Cortes \& Vapnik, 1995; Osuna, Freund, \& Girosi, 1997). However, SVMs require to solve a quadratic optimization problem which needs resources that are at least quadratic on the number of training examples, and it is thus hopeless to try solving problems having millions of examples using classical SVMs.

In order to overcome this drawback, we propose in this paper to use a mixture of several SVMs, each of them trained only on a part of the dataset. The idea of an SVM mixture is not new, although previous attempts such as Kwok's paper on Support Vector Mixtures (Kwok, 1998) did not train the SVMs on part of the dataset but on the whole dataset and hence could not overcome the time complexity problem for large datasets. We propose here a simple method to train such a mixture, and we will show that in practice this method is much faster than training only one SVM, and leads to results that are at least as good as one $S V M$. We conjecture that the training time complexity of the proposed approach with respect to the number of examples is sub-quadratic for large data sets. Moreover this mixture can be easily parallelized, which could improve again significantly the training time.

The organization of the paper goes as follows: in the next section, we briefly introduce the SVM model for classification. In section 3 we present our mixture of SVMs, followed in section 4 by some comparisons to related models. In section 5 we show some experimental results, first on a toy dataset, then on a large real-life dataset. A short conclusion then follows.

\section{Introduction to Support Vector Machines}

Support Vector Machines (SVMs) (Vapnik, 1995) have been applied to many classification problems, generally yielding good performance compared to other algorithms. The decision function is of the form

$$
y=\operatorname{sign}\left(\sum_{i=1}^{N} y_{i} \alpha_{i} K\left(\boldsymbol{x}, \boldsymbol{x}_{i}\right)+b\right)
$$

where $\boldsymbol{x} \in \mathbb{R}^{d}$ is the d-dimensional input vector of a test example, $y \in\{-1,1\}$ is a class label, $\boldsymbol{x}_{i} \in \mathbb{R}^{d}$ is the input vector for the $i^{t h}$ training example, $N$ is the number of training examples, $K\left(\boldsymbol{x}, \boldsymbol{x}_{i}\right)$ is a positive definite kernel function, and $\boldsymbol{\alpha}=\left\{\alpha_{1}, \ldots, \alpha_{N}\right\}$ and $b$ are the parameters of the model. Training an SVM consists in finding $\boldsymbol{\alpha}$ that minimizes the objective function

$$
Q(\boldsymbol{\alpha})=-\sum_{i=1}^{N} \alpha_{i}+\frac{1}{2} \sum_{i=1}^{N} \sum_{j=1}^{N} \alpha_{i} \alpha_{j} y_{i} y_{j} K\left(\boldsymbol{x}_{i}, \boldsymbol{x}_{j}\right)
$$

subject to the constraints

$$
\sum_{i=1}^{N} \alpha_{i} y_{i}=0
$$

and

$$
0 \leq \alpha_{i} \leq C \forall i
$$

The kernel $K\left(\boldsymbol{x}, \boldsymbol{x}_{i}\right)$ can have different forms, such as the Radial Basis Function (RBF):

$$
K\left(\boldsymbol{x}_{i}, \boldsymbol{x}_{j}\right)=\exp \left(\frac{-\left\|\boldsymbol{x}_{i}-\boldsymbol{x}_{j}\right\|^{2}}{\sigma^{2}}\right)
$$


with parameter $\sigma$.

Therefore, to train an SVM, we need to solve a quadratic optimization problem, where the number of parameters is $N$. This makes the use of SVMs for large datasets difficult: computing $K\left(x_{i}, x_{j}\right)$ for every training pair would require $O\left(N^{2}\right)$ computation, and solving may take up to $O\left(N^{3}\right)$. Note however that current state-of-the-art algorithms appear to have training time complexity scaling much closer to $O\left(N^{2}\right)$ than $O\left(N^{3}\right)$ (Collobert \& Bengio, 2001).

\section{Mixtures of SVMs}

In this section we introduce a new type of mixture of SVMs. The proposed model should minimize the following cost function:

$$
C=\sum_{i=1}^{N}\left[h\left(\sum_{m=1}^{M} w_{m}\left(\boldsymbol{x}_{i}\right) s_{m}\left(\boldsymbol{x}_{i}\right)\right)-y_{i}\right]^{2}
$$

where $M$ is the number of experts in the mixture, $s_{m}\left(\boldsymbol{x}_{i}\right)$ is the output of the $m^{t h}$ expert given input $\boldsymbol{x}_{i}, w_{m}\left(\boldsymbol{x}_{i}\right)$ is the weight for the $m^{t h}$ expert given by a "gater" module taking also $\boldsymbol{x}_{i}$ in input, and $h$ is a transfer function which could be for example the hyperbolic tangent for classification tasks. Here each expert is an SVM, and we took a neural network for the gater in our experiments.

To train this model, we propose a very simple algorithm:

1. Divide the training set into $M$ random subsets of size near $N / M$.

2. Train each expert separately over one of these subsets.

3. Keeping the experts fixed, train the gater to minimize the cost (6) on the whole training set.

4. Reconstruct $M$ subsets: for each example $\left(\boldsymbol{x}_{i}, y_{i}\right)$,

- sort the experts in descending order according to the values $w_{m}\left(\boldsymbol{x}_{i}\right)$,

- assign the example to the first expert in the list which has less than $(N / M+c)$ examples ${ }^{1}$, in order to ensure a balance between the experts.

5. If a termination criterion is not fulfilled (such as a given number of iterations or a validation error going up), goto step 2.

Note that step 2 of this algorithm can be easily implemented in parallel as each expert can be trained separately on a different computer. Note also that step 3 can be an approximate minimization (as usually done when training neural networks).

\section{Related Models}

The idea of mixture models is quite old and has given rise to very popular algorithms, such as the well-known Mixture of Experts (Jacobs, Jordan, Nowlan, \& Hinton, 1991) where the cost function is similar to equation (6) but where the gater and the experts are trained, using gradient descent or EM, on the whole dataset (and not subsets) and their parameters are trained simultaneously. Hence such an algorithm is quite demanding in terms of resources when the dataset is large, if training time scales like $O\left(N^{p}\right)$ with $p>1$.

In the more recent Support Vector Mixture model (Kwok, 1998), the author shows how to replace the experts (typically neural networks) by SVMs and gives a learning algorithm for this model. Once again the resulting mixture is trained jointly on the whole dataset, and hence does not solve the quadratic barrier when the dataset is large.

In another divide-and-conquer approach (Rida, Labbi, \& Pellegrini, 1999), the authors propose to first divide the training set using an unsupervised algorithm to cluster the data (typically a mixture

\footnotetext{
${ }^{1}$ where $c$ is a small positive constant
} 
of gaussians), then train an expert (such as an SVM) on each subset of the data corresponding to a cluster, and finally recombine the outputs of the experts. Here, the algorithm does indeed train separatly the experts on small datasets, like the present algorithm, but there is no notion of a loop reassigning the examples to experts according to the prediction made by the gater of how well each expert performs on each example. Our experiments suggest that this element is essential to the success of the algorithm.

\section{$5 \quad$ Experiments}

In this section, we present two sets of experiments comparing the new mixture of SVMs to other machine learning algorithms.

\subsection{A Toy Problem}

In the first series of experiments, We first tested the mixture on an artificial toy problem where we generated 10000 training examples and 10000 test examples. The problem had two non linearly separable classes and had two input dimensions. On Figure 1 we show the decision surfaces obtained first by a linear SVM, then by a gaussian SVM, and finally by the proposed mixture of SVMs. Moreover, in the latter, the gater was a simple linear function and there were two linear SVMs in the mixture. This artificial problem thus shows clearly that the algorithm seems to work, and is able to combine, even linearly, very simple models in order to produce a non-linear decision surface.

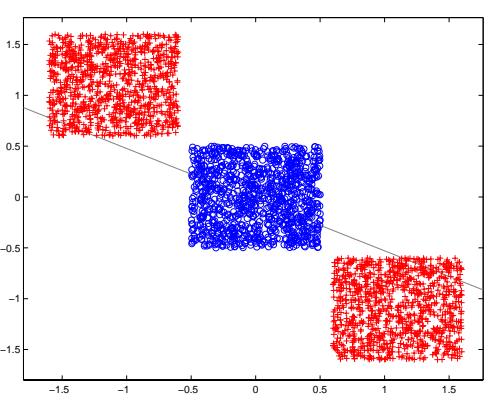

(a) Linear SVM

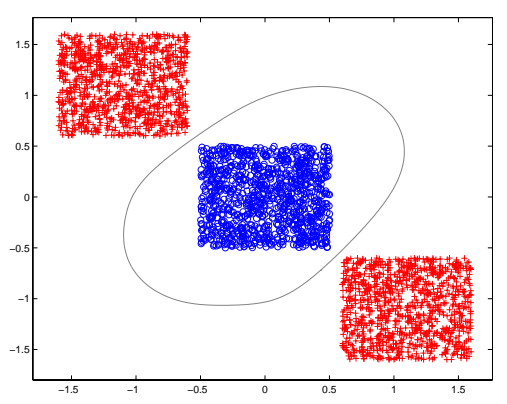

(b) Gaussian SVM

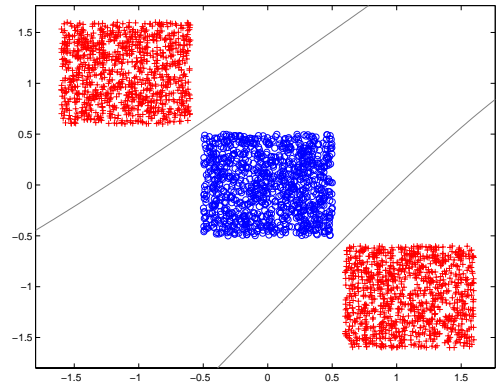

(c) Mixture of two linear SVMs

Figure 1: Comparison of the decision surfaces obtained by (a) a linear SVM, (b) a gaussian SVM, and (c) a linear mixture of two linear SVMs, on a two-dimensional classification toy problem.

\subsection{A Large-Scale Realistic Problem: Forest}

For a more realistic problem, we did a series of experiments on part of the UCI Forest dataset ${ }^{2}$. Since this is a classification problem with 7 classes, we modified it in a binary classification problem where the goal was to separate class 2 from the other 6 classes. The dataset had more than 500000 examples and this enabled us to prepare a series of experiments as follows:

- We kept a test set of 50000 examples to compare the best mixture of SVMs to other learning algorithms.

- We used a validation set of 10000 examples to select the best mixture of SVMs, varying the number of experts and the number of hidden units in the gater.

\footnotetext{
${ }^{2}$ The Forest dataset is available on the UCI website at the following address: ftp://ftp.ics.uci.edu/pub/machine-learning-databases/covtype/covtype.info.
} 
- We trained our models on different training sets, using from 100000 to 400000 examples.

- The mixtures had from 10 to 50 expert SVMs with gaussian kernel and the gater was an MLP with between 25 and 500 hidden units.

Note that since the number of examples was quite large, we selected the internal training parameters such as the $\sigma$ of the gaussian kernel of the SVMs or the learning rate of the gater using a held-out portion of the training set. We compared our models to

- a single MLP, where the number of hidden units was selected by cross-validation between 25 and 250 units,

- a single SVM, where the parameter of the kernel was also selected by cross-validation,

- a mixture of SVMs where the gater was replaced by a constant vector, assigning the same weight value to every expert.

Table 1 gives the results of a first series of experiments with a fixed training set of 100000 examples. Note that we did not select among the variants of the gated SVM mixture using only performance over the validation set but also taking into account the time to train the model. The selected model had 50 experts and a gater with 150 hidden units. A model with 500 hidden units would have given a performance of $8.1 \%$ over the test set but would have taken 621 minutes on one machine (and 388 minutes on 50 machines).

\begin{tabular}{|c|c|c|c|c|c|c|}
\hline \multirow[b]{3}{*}{ 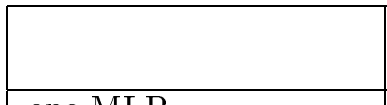 } & \multirow{2}{*}{\multicolumn{3}{|c|}{$\begin{array}{ccc}\text { Train Valid } & \text { Test } \\
& \text { Error }(\%)\end{array}$}} & \multicolumn{2}{|c|}{ Time (minutes) } & \multirow{2}{*}{$\begin{array}{l}\text { Total } \\
\text { \# SV }\end{array}$} \\
\hline & & & & $(1 \mathrm{cpu})$ & $(50 \mathrm{cpu})$ & \\
\hline & 17.56 & 18.12 & 18.15 & 12 & & 100 \\
\hline & 16.0 & 16.68 & 16.76 & 3231 & & 42451 \\
\hline unifo & 19.6 & 19.90 & 20.31 & 85 & 2 & 52846 \\
\hline gated & 5.91 & 8.90 & 9.28 & 237 & 73 & 31703 \\
\hline
\end{tabular}

Table 1: Comparison of performance between an MLP (100 hidden units), a single SVM, a uniform SVM mixture where the gater always output the same value for each expert, and finally a mixture of SVMs as proposed in this paper.

As it can be seen, the gated SVM outperformed all models in terms of training, validation, and test error. It was also much faster, even on one machine, than the SVM and since the mixture could easily be parallelized (each expert can be trained separately), we also gave the time it took to train on 50 machines. It is also interesting to note that the total number of support vectors of the gated SVM was less than the number of support vectors of the SVM. In a first attempt to understand these results, one can at least say that the power of the model does not lie only on the gater, since a single MLP was pretty bad, it is neither only because we used SVMs, since a single SVM was not as good as the gated mixture, and it was not only because we divided the problem into many sub-problems since the uniform mixture also performed badly. It seems to be a combination of all these elements.

We also did a series of experiments in order to see the influence of the number of hidden units of the gater as well as the number of experts in the mixture. Figure 2 shows the validation error of different mixtures of SVMs, where the number of hidden units varied from 25 to 500 and the number of experts varied from 10 to 50 . There is a clear performance improvement when the number of hidden units is increased, while the improvement with additional experts exists but is less clear. Note however that the training time increases also rapidly with the number of hidden units while it slightly decreases with the number of experts if one uses one computer per expert.

In order to find how the algorithm scaled with respect to the number of examples, we then compared the same mixture of experts (50 experts, 150 hidden units in the gater) on different training set sizes. Table 3 shows the training time of the mixture of SVMs trained on training sets of sizes from 100000 
Validation error as a function of the number of hidden units of the gater and the number of experts

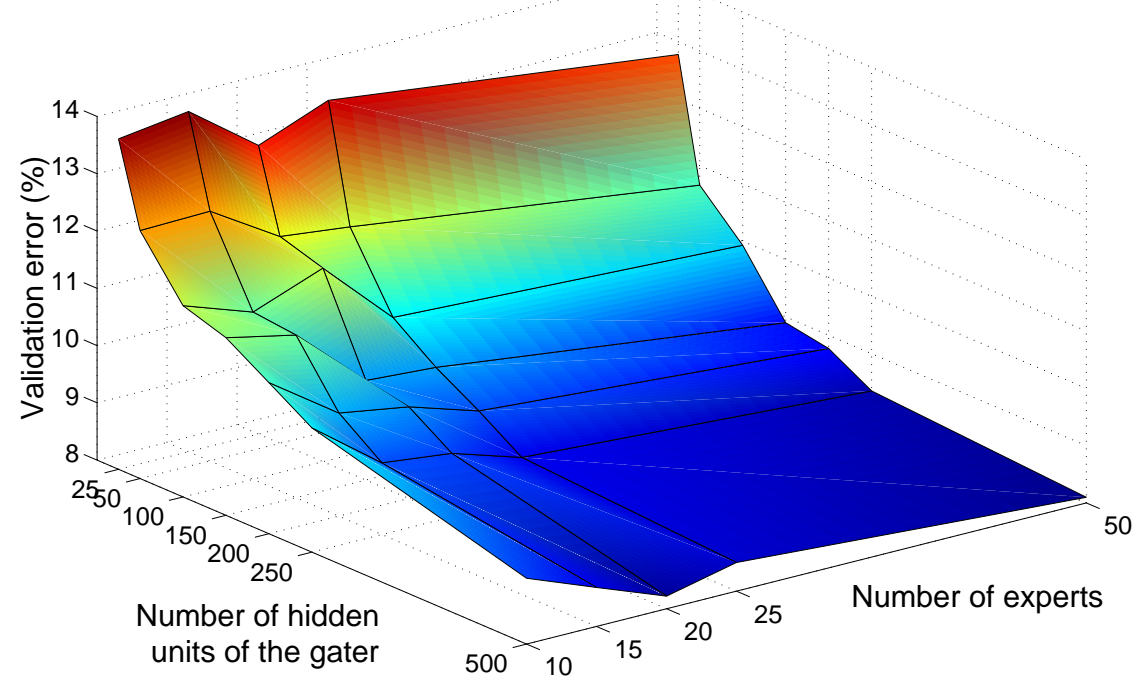

Figure 2: Comparison of the validation error of different mixtures of SVMs with various number of hidden units and experts.

to 400000 . It seems that, at least in this range and for this particular dataset, the mixture of SVMs scales linearly with respect to the number of examples, and not quadratically as a classical SVM. It is interesting to see for instance that the mixture of SVMs was able to solve a problem of 400000 examples in less than 7 hours (on 50 computers) while it would have taken more than one month to solve the same problem with a single SVM.

Finally, figure 4 shows the evolution of the training and validation errors of a mixture of 50 SVMs gated by an MLP with 150 hidden units, during 5 iterations of the algorithm. This should convince that the loop of the algorithm is essential to obtain good performance.

\section{Conclusion}

In this paper we have presented a new algorithm to train a mixture of SVMs that gave very good results compared to classical SVMs either in terms of training time, generalization performance, or sparseness. Moreover, the algorithm appears to scale linearly with the number of examples, at least between 100000 and 400000 examples. Furthermore, the proposed algorithm has also been tested on another task with a similar number of examples and also yielded performance improvements.

These results are extremely encouraging and suggest that the proposed method could allow training SVM-like models for very large multi-million data sets in a reasonable time. If training of the neural network gater with stochastic gradient takes time that grows much less than quadratically, as we conjecture it to be the case for very large data sets (to reach a "good enough" solution), then the whole method is clearly sub-quadratic in training time with respect to the number of training examples.

\section{Acknowledgments}

RC would like to thank the Swiss National Science Foundation for financial support (project FN2100061234.00). YB would like to thank the NSERC funding agency and $\mathrm{NCM}^{2}$ network for support. 


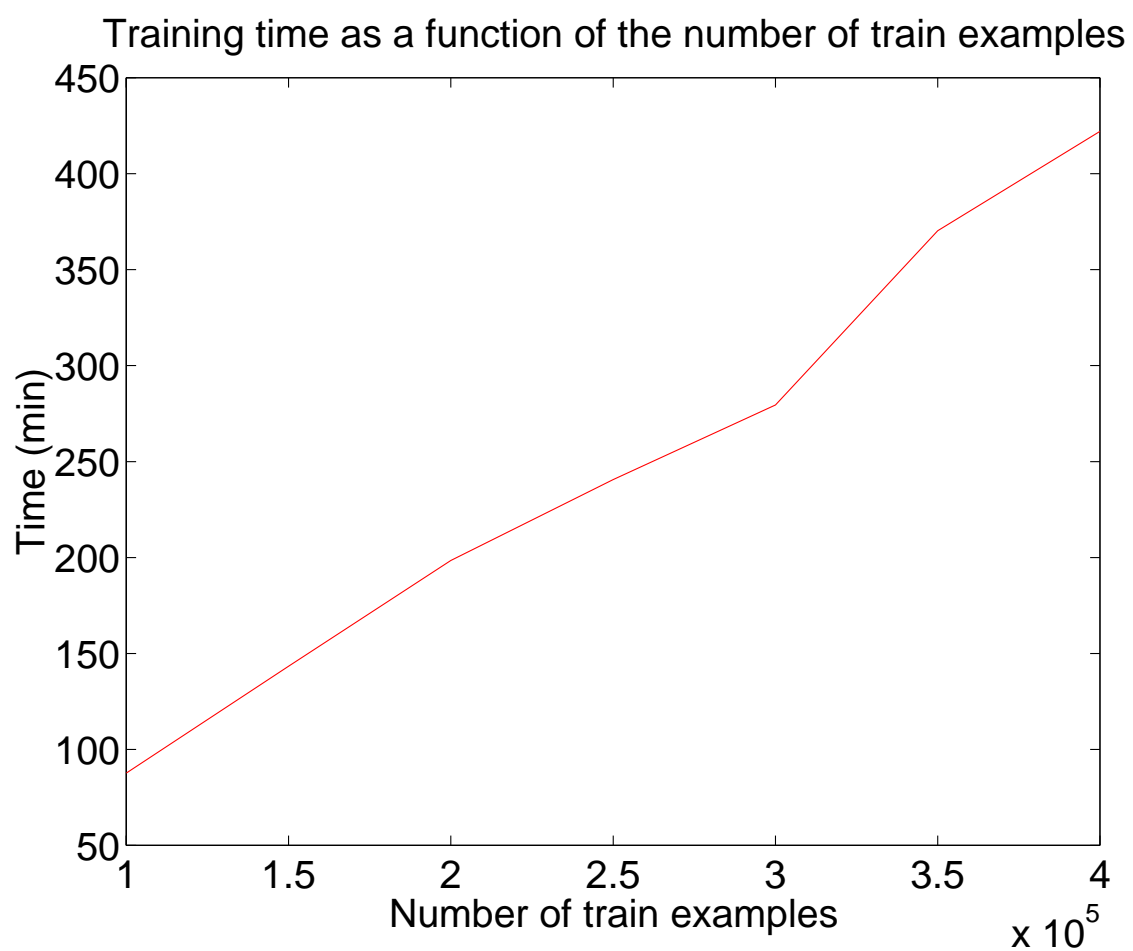

Figure 3: Comparison of the training time of the same mixture of SVMs (50 experts, 150 hidden units in the gater) trained on different training set sizes, from 100000 to 400000.

\section{References}

Collobert, R., \& Bengio, S. (2001). SVMTorch: Support vector machines for large-scale regression problems. Journal of Machine Learning Research, 1, 143-160.

Cortes, C., \& Vapnik, V. (1995). Support vector networks. Machine Learning, 20, 273-297.

Jacobs, R. A., Jordan, M. I., Nowlan, S. J., \& Hinton, G. E. (1991). Adaptive mixtures of local experts. Neural Computation, 3(1), 79-87.

Kwok, J. T. (1998). Support vector mixture for classification and regression problems. In Proceedings of the international conference on pattern recognition (icpr) (pp. 255-258). Brisbane, Queensland, Australia.

Osuna, E., Freund, R., \& Girosi, F. (1997). Training support vector machines: an application to face detection. In Ieee conference on computer vision and pattern recognition (pp. 130-136). San Juan, Puerto Rico.

Rida, A., Labbi, A., \& Pellegrini, C. (1999). Local experts combination trough density decomposition. In International workshop on ai and statistics (uncertainty'99). Morgan Kaufmann.

Vapnik, V. N. (1995). The nature of statistical learning theory (second ed.). Springer. 


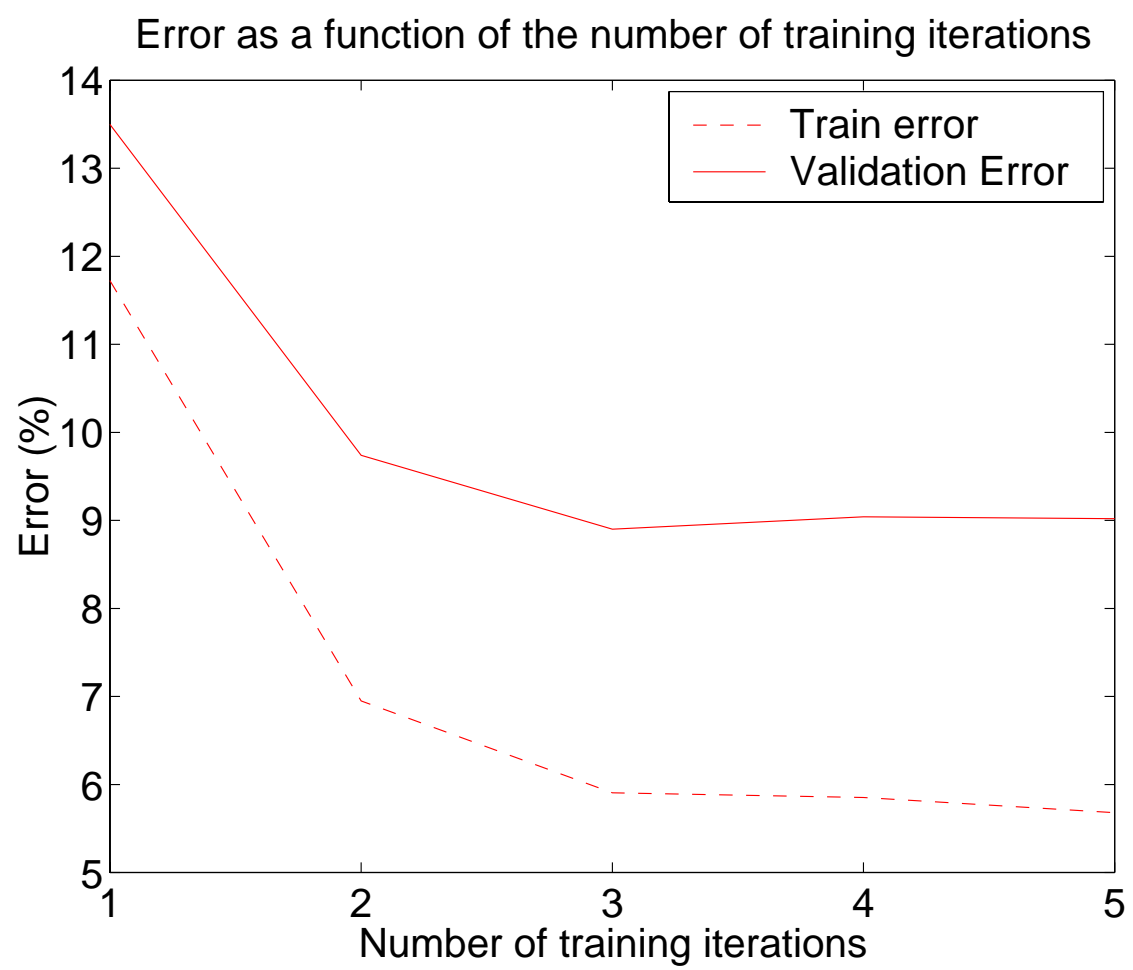

Figure 4: Comparison of the training and validation errors of the mixture of SVMs as a function of the number of training iterations. 